\title{
Flexible Representative Democracy: An Introduction with Binary Issues
}

\author{
Ben Abramowitz ${ }^{1}$ and Nicholas Mattei ${ }^{2}$ \\ ${ }^{1}$ Rensselaer Polytechnic Institute, Troy, NY, USA \\ ${ }^{2}$ Tulane University, New Orleans, LA, USA \\ abramb@rpi.edu,nsmattei@tulane.edu
}

\begin{abstract}
We introduce Flexible Representative Democracy (FRD), a novel hybrid of Representative Democracy $(\mathrm{RD})$ and Direct Democracy $(\mathrm{DD})$, in which voters can alter the issue-dependent weights of a set of elected representatives. In line with the literature on Interactive Democracy, our model allows the voters to actively determine the degree to which the system is direct versus representative. However, unlike Liquid Democracy, FRD uses strictly non-transitive delegations, making delegation cycles impossible, preserving privacy and anonymity, and maintaining a fixed set of accountable elected representatives. We present FRD and analyze it using a computational approach with issues that are independent, binary, and symmetric; we compare the outcomes of various democratic systems using Direct Democracy with majority voting and full participation as an ideal baseline. We find through theoretical and empirical analysis that FRD can yield significant improvements over RD for emulating DD with full participation.
\end{abstract}

\section{Introduction}

Since the Athenian Ecclesia in 595 BCE Direct Democracy (DD) as an ideal collective decision making scheme has loomed large in the western imagination [Dunn, 1995]. While DD may be desirable it becomes impractical at scale as it places too much burden on individual decisions makers: everyone must be well-informed on every issue and available to vote [Green-Armytage, 2015]. In addition to the attention requirements, voters are also required to know and be able to articulate their preferences at the time of every vote. While preferences and preference learning are large research areas in AI [Domshlak et al., 2011] every voter may not have enough knowledge, information, time, energy, or incentive to participate, particularly when issues are numerous or complex.

Given the prohibitive costs of implementing a large-scale DD in both human and agent societies, we often resort to representation, relying upon a set of proxies to decide on the voters' behalf. Countries have parliaments, companies have elected boards, and groups of agents select leaders to represent them [Yu et al., 2010]. Sets of representatives have been used in many contexts and disciplines to reduce the computation and communication burden of decision makers. The Computational Social Choice (COMSOC) community [Brandt et al., 2016] has produced a large body of research on how to select and weight representatives. Indeed, using multi-winner voting [Skowron et al., 2016], we can view the winners as a set of exemplars that may be used to decide some downstream application.Often it is beneficial to elect fixed committees which meet certain axiomatic criteria. For example, committees should be proportional and have justified representation of the voters [Aziz et al., 2017]. Intuitively, these difficulties in electing committees carry through to the setting of Representative Democracy (RD) where the committee makes decisions in the interest of the voters/agents who elect them. This setting was studied by Skowron [2015] who proved that when we want to optimize for the sum of voters who are represented on each issue the $k$-Median rule optimal.

Since DD can be impractical and RD comes with inherent tradeoffs and limitations, hybridizations of the two have arisen under the umbrella of Interactive Democracy. Coupled with modern communication technologies, a large number of proposed democratic decision making systems have been proposed, and Interactive Democracy has become an important area of research and application for AI [Brill, 2018]. Perhaps the most popular version of this today is Liquid Democracy (LD); which has received significant attention in the political science [Green-Armytage, 2015], AI [Kahng et al., 2018] and agents communities [Brill and Talmon, 2018], and has been implemented in both corporate [Hardt and Lopes, 2015] and political settings [Blum and Zuber, 2016].

In contrast to existing proposals, our model of Flexible Representative Democracy (FRD) maintains a set of expert representatives while allowing voters to guarantee their own representation without raising the voters' minimum required burden. In an FRD voters elect a set of representatives to serve a term during which they decide the outcomes over a set of issues. Each voter, by default, allocates a fraction of their voting power to each member of the committee. If this allocation is uniform and we stop here, we are left with the traditional model of RD where each representative has equal power. However, for each issue under consideration in FRD, the voters may deviate from this default by delegating their voting power to any subset of the committee. If all voters use their option to delegate on each issue, as long as there is at 
least one representative who agrees with each voter's view, the outcome perfectly recovers DD. Voters have both the election and the flexible delegation option as tools for achieving representation and holding representatives accountable.

In an FRD, voters have great flexibility in determining how they are represented and the mandated disclosure of representatives' votes guarantees that an attentive voter can be fully informed about how their voting power will be and was used. For example, the day after the election an inattentive voter might choose a few elected representatives they trust, apportion the power of their vote to these few for all future issues and pay no attention until the next election. A more attentive voter might alter their allocations on an issue-by-issue basis as issues arise, reacting to representatives' votes. In general, voters determine the granularity with which they privately express their preferences over issues via the representatives. In addition, in an FRD voters may or may not be permitted to vote directly on the issues, depending upon the application.

Contributions. We introduce Flexible Representative Democracy (FRD), a new model of Interactive Democracy which transitions, at the discretion of the voters, between $\mathrm{RD}$ and DD. FRD solves standing issues in the literature on Interactive Democracy including maintaining a fixed, elected committee to generate legislation, making delegation cycles impossible, and preserving voter anonymity. We analyze our model theoretically using independent, binary, symmetric issues. We show that electing an optimal set of representatives is hard for any large-scale RD no matter the voting rule, thus motivating the use of flexible delegations. Thus, we demonstrate the theoretical ability of delegations under FRD to overcome the limitations of RD, providing empirical results demonstrating that FRD outperforms both $\mathrm{RD}$ and Proxy Voting for representing the majority will.

\section{Model and Preliminaries}

We primarily consider three democratic decision systems: Direct Democracy (DD), Representative Democracy (RD), and our model: Flexible Representative Democracy (FRD). Given a set of voters $\mathcal{V}$ with preferences over the alternatives for each issue in a set of issues $\mathcal{S}$, we represent their collective preferences by a preference profile $\mathcal{P}_{\mathcal{V} \mathcal{S}}$. In DD, a decision rule $\mathcal{R}_{\mathcal{S}}$ takes the $\mathcal{P}_{\mathcal{V} \mathcal{S}}$ as input maps them to a set of outcomes over the issues $\mathcal{O}_{D D} ; \mathcal{R}_{\mathcal{S}}\left(\mathcal{P}_{\mathcal{V S}}\right) \rightarrow \mathcal{O}_{D D}{ }^{1}$

By contrast, in RD voters' preferences on the issues may never be directly elicited. Rather, voters report their preferences over a set of candidates $\mathcal{C}$. We denote the collective preferences of the voters over the candidates by the electoral profile $\mathcal{P}_{\mathcal{V C}}$. An election rule $\mathcal{R}_{\mathcal{E}}$ (i.e. multi-winner voting rule) is then used to aggregate these preferences and select a subset of candidates $\mathcal{D}$ to serve as representatives, $\mathcal{R}_{\mathcal{E}}\left(\mathcal{P}_{\mathcal{V C}}\right) \rightarrow \mathcal{D} \subseteq \mathcal{C}$. In a standard $\mathrm{RD}$, a decision rule is then applied to the public preferences of the representatives over the issues $\mathcal{P}_{\mathcal{D S}}$ to determine the set of outcomes on all issues $\mathcal{O}_{R D} ; \mathcal{R}_{\mathcal{S}}\left(\mathcal{P}_{\mathcal{D S}}\right) \rightarrow \mathcal{O}_{R D}$. Clearly, RD may produce different outcomes than DD, and may leave accessible information about voter preferences unsolicited and unused.

\footnotetext{
${ }^{1}$ More generally, different decision rules could be used on different issues within a single DD, RD, or FRD.
}

In FRD, as with RD, the voters elect a set of representatives $\mathcal{R}_{\mathcal{E}}\left(\mathcal{P}_{\mathcal{V C}}\right) \rightarrow \mathcal{D} \subseteq \mathcal{C}$. However, for every issue, a divisible unit of voting power is given to each voter rather than to each representative. Automatically after the election, the voters' issue-specific votes are distributed among the representatives according to some default distribution mechanism. Subsequently, every voter has the option to alter how their voting power is assigned to the representatives and may change this for any subset issues at once or on an issue-by-issue basis. We refer to this process of deviating from the default and actively allocating voting power to representatives as delegation. Delegations are not permanent and may be altered before an issue is decided. We let $W_{j l}^{i}$ represent the voting power allocated by voter $v_{j} \in \mathcal{V}$ to candidate $c_{l} \in \mathcal{C}$ on issue $s^{i} \in \mathcal{S}$, yielding a collective matrix of weights $W$. In FRD, a decision rule $\mathcal{R}_{\mathcal{S}}$ is then applied to the representatives' preferences taking these weights into account $\mathcal{R}_{\mathcal{S}}\left(W, \mathcal{P}_{\mathcal{D S}}\right) \rightarrow \mathcal{O}_{F R D}$. If voters have the option to vote directly on issues rather than having their voting power only distributed to representatives (e.g., more similar to LD), $W$ and $\mathcal{P}_{\mathcal{D S}}$ can be augmented to allow voters to "delegate" to themselves.

\subsection{Model Specification}

We restrict our attention to a simple type of FRD. Our objective is to compare the extent to which RD and FRD can emulate DD, which we hold as "optimal." We consider a setting with symmetric, binary issues so each issue $s^{i} \in \mathcal{S}$ has two possible outcomes $\mathcal{O}^{i} \in\{0,1\}$, and there are $2^{r}$ possible outcome vectors over $|\mathcal{S}|=r$ of the form $\mathcal{O} \in\{0,1\}^{r}$. We assume all issues are independent, this is a simplifying assumption that circumvents issues raised in judgment aggregation [List and Puppe, 2009], though an important direction for future work. Without loss of generality, we label each of the alternatives preferred by the (weak) majority of voters 1 and the other 0 , breaking ties randomly (when $N$ is even). Thus, the ideal majoritarian outcome over the issues is $\{1\}^{r}$.

Each voter in the set of voters $\mathcal{V}=\left\{v_{1}, \ldots, v_{N}\right\}$ has a preferred alternative $v_{j}^{i} \in\{0,1\}$ for every issue $s^{i}$ in the set of issues $\mathcal{S}=\left\{s^{1}, \ldots, s^{r}\right\}$. We let vector $\vec{v}_{j}=\left\{v_{j}^{1}, \ldots, v_{j}^{r}\right\}$ represent the preferred outcome of voter $v_{j}$ over the issues, resulting in the collective profile $\mathcal{P}_{\mathcal{V} \mathcal{S}}=\left\{\vec{v}_{j}: v_{j} \in \mathcal{V}\right\}$. Similarly, when we have representatives, we represent the preference profile of the candidates as $\mathcal{P}_{\mathcal{C S}}=\left\{\vec{c}_{l}: c_{l} \in \mathcal{C}\right\}$ where candidate $c_{l}$ has preferred outcome $\vec{c}_{l} \in\{0,1\}^{r}$.

We define the agreement between any two outcome vectors $\mathcal{O}_{1}, \mathcal{O}_{2}$ of length $r$ as $L\left(\mathcal{O}_{1}, \mathcal{O}_{2}\right)=1-\frac{1}{r} \sum_{i=1}^{r}\left|\mathcal{O}_{1}^{i}-\mathcal{O}_{2}^{i}\right|$, i.e., the fraction of issues for which the outcomes are the same. When comparing to an ideal set of outcomes (i.e. $\left.\mathcal{O}_{D D}\right)$, this is the number of issues decided "correctly." We will often refer to the agreement between a voter and candidate $L\left(\vec{v}_{j}, \vec{c}_{l}\right)$ as well as the agreement between the outcomes of different democratic systems, i.e. $\mathrm{L}\left(\mathcal{O}_{D D}, \mathcal{O}_{R D}\right)$.

We consider three possible ways voters might express their preferences over the candidates: approvals, total orderings, and normalized weights. We make an assumption about these preferences to give RD the greatest chance of maximizing $L\left(\mathcal{O}_{D D}, \mathcal{O}_{R D}\right)$ : we assume each voters' preferences over the candidates are induced by their agreement. When voters sub- 
mit approval ballots, we assume $v_{j}$ approves of $c_{l}$ if and only if $L\left(\vec{v}_{j}, \vec{c}_{l}\right)>1 / 2$. When voters report total orderings $\left(\succ_{j}\right)$, we assume they order all candidates so that $c_{l} \succ_{j} c_{h}$ only if $L\left(v_{j}, c_{l}\right) \geq L\left(v_{j}, c_{h}\right)$ where ties are broken privately (e.g. randomly). When voters report their preferences as normalized weights, $w_{j}^{l}=L\left(v_{j}, c_{l}\right) / \sum_{c_{h} \in C} L\left(v_{j}, c_{h}\right)$.

For DD, we only consider the simple majority rule as our decision rule $\mathcal{R}_{\mathcal{S}}$ as our issues are binary and symmetric. For our representative systems we compare several common, anonymous election rules $\mathcal{R}_{\mathcal{E}}$ with a fixed, odd committee size $k$, i.e., $\mathcal{D} \subseteq \mathcal{C}$ where $|\mathcal{D}|=k$. All rules considered in our simulations are deterministic other than randomized tie-breaking. In the setting where voters submit approval ballots, we consider Approval Voting and Reweighted Approval Voting (AV, RAV). When voters submit their preferences over candidates as total orderings, we consider Single-Transferrable Vote (STV), Borda, $k$-Median, and Chamberlin-Courant (CC). When voters submit their ballots as normalized weights over the candidates, we consider the rule which selects the $k$ candidates who receive the largest total weight. Lastly, we compare these rules to selecting $k$ representatives uniformly at random from the candidates. We refer the reader to Brandt et al. [2016] and Skowron [2015] for complete definitions. We assume that the preferences of candidates do not change before or after they are elected as representatives. Without loss of generality, let the winning candidates be the lowest $k$ indexed $\left\{c_{1}, \ldots, c_{k}\right\}$ such that representative $d_{l} \in \mathcal{D}$ is candidate $c_{l}$ and $\vec{d}_{l}=\overrightarrow{c_{l}}$.

Given a set $\mathcal{D}$ of $k$ representatives, we want to evaluate the capability of this set to represent the majority will of the voters, i.e., recover the outcome of DD with full voter turnout. Hence, we define coverage and majority agreement as metrics to evaluate these systems. Let $k_{1}^{i}$ and $k_{0}^{i}$ represent the number of representatives who prefer alternatives 1 and 0 on issue $s^{i}$ respectively, such that $k_{1}^{i}+k_{0}^{i}=k$.

- The majority agreement on a set of issues is the fraction of issues on which the majority of representatives agree with the majority of voters $\left(k_{1}^{i}>\frac{k}{2}>k_{0}^{i}\right)$. This quantity is $\frac{1}{r} \sum_{i \in\{1, \ldots, r\}} L\left(\mathcal{O}_{R D}^{i}, \mathcal{O}_{D D}^{i}\right)$.

- The coverage of a set of issues is the fraction of issues covered on which at least one representative agrees with the voter majority $\left(0<k_{1}^{i}\right)$.

We allocate each voter one divisible vote for each independent issue, maintaining the principle of "one person, one vote." By default, each voter's unit of voting power is distributed uniformly among the representatives on each issue so $W_{l}^{i}=N / k$ initially for each of the $k$ candidates and $r$ issues (exactly as in RD). However, various distributions from the literature on voting power [Shapley and Shubik, 1954; Banzhaf III, 1964] and Proxy Voting [Alger, 2006] are worth consideration in the future. We do not consider abstentions by representatives nor voter, i.e., a voter assigns less than a full vote across representatives, and we do not permit voters to vote directly on the issues. Hence, the total voting power held by the representatives remains $N$ collectively for all issues.

For our purposes, the total weight $W_{l}^{i}$ assigned to representative $d_{l}$ on issue $s^{i}$ is the sum of the voting power they receive from default and delegation, $\sum_{j} W_{j l}^{i}$. Consequently, the total weight assigned to representatives who agree with the voter majority is $X_{1}^{i}=\sum_{d_{l}^{i}=1} W_{l}^{i}$. In this paper our decision rule for FRD is weighted majority with random tie breaking: $\mathcal{O}^{i}=1$ if $X_{1}^{i}>N / 2, \mathcal{O}^{i}=0$ if $X_{1}^{i}<N / 2$, and $\mathcal{O}^{i}=1$ with probability $1 / 2$ if $X_{1}^{i}=N / 2$.

We assume that all voters are incisive on all issues. A voter is incisive if they only delegate voting power to representatives who agree with their preferred alternative. Similarly, we refer to delegations as being incisive if they exhibit this property on an issue. We relax the assumption of incisive voters in our simulations and consider voters who delegate only to their most preferred candidate(s) or divide their delegation evenly across their approved set. Relaxing these assumptions are important directions for future work discussed in the full version of this paper [Abramowitz and Mattei, 2018].

Example 1. Consider an FRD instance with issues $s^{1}$ and $s^{2}$, three voters, and three representatives. Below, the solid arrows from voter to representative indicate delegations, and any voter without an arrow defaults on that issue. The voter and representative preferences are given in the tables above and below the agents; both delegations are incisive.

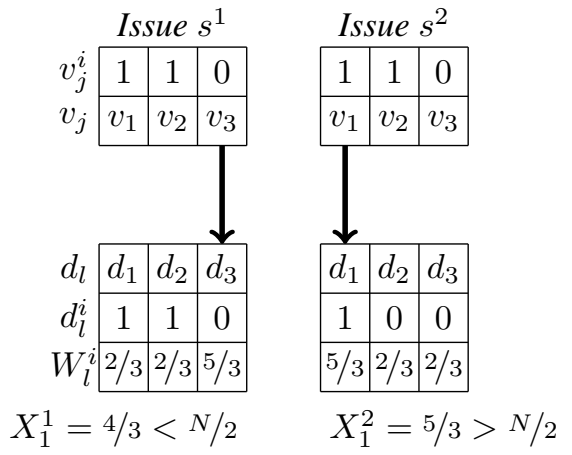

On issue $s^{1}$, the representative majority agrees with the voter majority, so $R D$ would yield $\mathcal{O}_{R D}^{1}=1$ as desired. However, since only the voter in the minority $\left(v_{3}^{1}=0\right)$ delegates, the weighted majority of representatives now decides the outcome in favor of the voter minority $\left(X_{1}^{1}<N / 2\right)$. Hence, FRD can make the outcomes worse than RD as measured against $D D$ in some cases. This can occur if the number of voters in the minority is large, the number of representatives who agree with the voter minority is large, and the voters in the minority delegate at a higher rate than the voters in the majority.

On issue $s^{2}$ the representative majority disagrees with the voter majority so the RD outcome (without delegations) would be $\mathcal{O}_{R D}^{1}=0$. Looking again at the figure we see the delegations flip the result to what would be achieved by $D D$ $\left(X_{1}^{2}>N / 2\right)$. Hence, FRD can improve the outcomes over $R D$ as measured against DD. Fortunately, for both issues, if any two or all of the voters delegate incisively, the outcome will always agree with the voter majority.

\section{Related Work}

Miller [1969], inspired by Tullock [1967] and the idea of shareholder proxy voting, suggested an interactive democratic system for legislation which could take place at scale using computers. Miller lamented the lack of flexibility in traditional Representative Democracy and sought to remedy this 
using a dynamic system of proxies, although he admitted this was not conducive to creating legislation. Soon after, [Shubik, 1970] warned that electronic systems may accelerate the legislative process in undesirable ways and suggested holding every referendum twice to guarantee time for sufficient public deliberation. Our use of a fixed, elected set of representatives answers Miller's question of how to produce legislation, and rather than holding redundant referenda we make representatives' votes public and then give the voters sufficient time to deliberate and alter their delegations.

Before the dawn of the internet, [Tullock, 1992] revisited his ideas from [Tullock, 1967] in a proposal that motivates the default and delegation mechanisms in FRD. The notion of the default distribution is similar to that proposed by Alger [2006], which suggests that the weights of representatives be based on the preferences of voters expressed in the election, but these weights are fixed during their term. By contrast, in FRD the weight of each representative on each issue is not strictly determined by the election. Cohensius et al. [2017] took an analytical approach to studying a Proxy Voting model close to that of Alger [2006] for decision making with no election, infinite voters, spatial preferences, and that agents lie in a metric space.

The hallmark of interactive democracies like FRD and Liquid Democracy is that rather than adjudicating whether a direct or representative system is better for achieving some objective and asserting it by fiat, the extent to which the system is direct or representative is itself a function of the "will of the voters". Currently, Liquid Democracy is the most well-known and well-studied form of Interactive Democracy, and has been studied from an algorithmic perspective as a decision-making process in the AI and COMSOC literature [Brill and Talmon, 2018; Kahng et al., 2018; Bloembergen et al., 2018; Christoff and Grossi, 2017] and elsewhere [Green-Armytage, 2015; Blum and Zuber, 2016; Brill, 2018; Hardt and Lopes, 2015]. ${ }^{2}$ Unlike Liquid Democracy, FRD does not allow transitive delegations nor delegations to another voter, thereby violating the second axiom proposed by Green-Armytage [2015]. Fractional delegations in FRD serve a similar function to that of the virtual committees proposed by Green-Armytage [2015], although in theory FRD could incorporate virtual committees as well as other mechanisms for delegating voting power.

The design of FRD is largely based on work in probabilistic voting, binary aggregation, statistical decision theory, and computational social choice. In particular, work on the optimal weighting of experts [Baharad et al., 2012; Nitzan and Paroush, 2017; Grofman and Feld, 1983; Nitzan and Paroush, 1982; Ben-Yashar and Nitzan, 1997], the Condorcet Jury Theorem [Grofman et al., 1983], variable electorates [Feld and Grofman, 1984; Smith, 1973; Paroush and Karotkin, 1989], and optimal committee sizes [Auriol and Gary-Bobo, 2012; Karotkin and Paroush, 2003; MagdonIsmail and Xia, 2018]. In FRD, one can view the voter delegations as a pseudo-tie breaking mechanism for the representatives or, conversely, see the default distribution as a way to dampen the variance in the outcome in that may oc-

\footnotetext{
${ }^{2}$ Also see B. Ford, Delegative Democracy at http://brynosaurus. com/deleg/deleg.pdf.
}

cur DD when the set of participating voters is small or biased. Another view is that electing representatives is analogous to a compression algorithm [Rodriguez and Steinbock, 2004], which is the algorithmic version of John Adams's alleged intuition that the representatives should be a microcosm of the population (taken from [Alger, 2006]). In this view, the delegations in FRD are analogous to a decompression mechanism where a higher delegation rate reduces the "loss" of representation. Our evaluations are similar to those of Skowron [2015], however, in their model the quality of the committee is measured as the sum of the voter proportion being represented for each issue, while we focus only on the total number of issues correct according to DD.

\section{Difficulties of Representative Democracy}

Electing good committees is hard. In fact, electing a set of representatives which maximizes majority agreement on binary issues is NP-Hard even if we know the view of every voter on every issue. The easier problem of maximizing coverage is also NP-Hard. We refer to the problems of selecting $k$ representatives to maximize coverage and majority agreement as Max k-Coverage and Max k-Majority Agreement, respectively. Note that if the majority view of the voters were known, coverage could be approximated deterministically in polynomial time within a factor of $1-1 / e$ by a greedy algorithm, and this bound is tight [Feige, 1998]. The proofs for our complexity results can be found in the full version of this paper [Abramowitz and Mattei, 2018].

Theorem 2. Max k-Coverage: If the candidates' preferences and the outcome preferred by the majority of voters are known for every issue, selecting the subset of candidates that maximizes the number of issues covered is NP-hard.

Proof. We show a reduction from the NP-Hard problem of MAX K-COVER [Garey and Johnson, 1979; Feige, 1998] to Max k-Coverage. In MAX K-COVER, given a set $U=$ $\left\{x_{1}, \ldots, x_{r}\right\}$ of $r$ points, a collection $Z=\left\{z^{1}, \ldots, z^{m}\right\}$ of subsets of $U$, and an integer $k$ we must select $k$ subsets from $Z$ such that their union has maximum cardinality. Given an instance $(U, Z, k)$ of MAX K-COVER we create an instance of Max k-Coverage as follows. For every point $x_{i} \in U$ create an issue $s^{i}$ and for every subset $z^{l} \in Z$ create a candidate $c_{l}$. For all points $x_{i}$ and subsets $z^{l}$, if $x_{i} \in z^{l}$ then let $c_{l}^{i}=1$, otherwise let $c_{l}^{i}=0$. Let $k$ be the number of representatives we will elect. There is a one-to-one correspondence between the number of issues covered by our $k$ representatives and the cardinality of the corresponding subsets in the original instance; any set of $k$ candidates that maximizes coverage corresponds exactly to a collection of $k$ subsets in our MAX K-COVER instance whose union has maximum cardinality.

The hardness of achieving coverage even in the unrealistic case where the voter majority is known for every issue supports the idea that voters should be able to vote directly when the issues are binary to ensure the majority will is always recoverable. Or, at the very least, a dummy candidate with no default power should be created automatically on any binary issue for which the representatives are unanimous. Note that adding such a candidate automatically can only improve the 
outcome, as it can only change the outcome of the majority of voters delegate to the dummy.

Theorem 3. Max k-Agreement: If the candidates' preferences and the outcome preferred by the majority of voters are known for every issue, selecting the subset of $k$ candidates whose majority agrees with the voter majority on the greatest number of issues is NP-hard.

Proof. We prove the hardness of Max k-Majority Agreement by polynomial-time reduction from our problem of Max k-Coverage. Given an instance of Max k-Coverage with input $\left(\mathcal{S}=\left\{s^{1}, \ldots, s^{r}\right\}, \mathcal{C}=\left\{c_{1}, \ldots, c_{m}\right\}, \mathcal{P}_{\mathcal{C S}}=\right.$ $\left.\left\{\overrightarrow{c_{1}}, \ldots, \overrightarrow{c_{m}}\right\}, k\right)$ we construct an instance of Max k-Majority Agreement with input $\left(\tilde{\mathcal{S}}, \tilde{\mathcal{C}}, \tilde{\mathcal{P}_{\mathcal{C} S}}, \tilde{k}\right)$ as follows. Create a set of binary issues $\tilde{\mathcal{S}}=\left\{\tilde{s}_{1}, \ldots, \tilde{s}_{2 r+1}\right\}$ and a set of candidates $\tilde{\mathcal{C}}=\left\{\tilde{c}_{1}, \ldots, \tilde{c}_{m+k+1}\right\}$. Let $\tilde{k}=2 k+1$. For $l \leq m$, $\tilde{c}_{l}^{i}=c_{l}^{i}$ for $i \leq r$ and $\tilde{c}_{l}^{i}=0$ for $r<i \leq 2 r+1$. For $m<l<m+k+1, \tilde{c}_{l}^{i}=1$ for all $i$. And for $l=m+k+1$, $\tilde{c}_{l}^{i}=0$ for $i \leq r$ and $\tilde{c}_{l}^{i}=1$ for $r<i \leq 2 r+1$.

$\tilde{\mathcal{D}}$ agrees with the voter majority on issues $\left\{s^{r+1}, \ldots, s^{2 r+1}\right\}$ iff $\tilde{\mathcal{D}}$ contains $\left\{\tilde{c}_{m+1}, \ldots, \tilde{c}_{m+k+1}\right\}$, because this is the only way at least $k+1$ out of the $2 k+1$ representatives can agree with the voter majority on any of these issues. Therefore any set $\tilde{\mathcal{D}} \subseteq \tilde{\mathcal{C}}$ of $2 k+1$ representatives which maximizes majority agreement must contain $\left\{\tilde{c}_{m+1}, \ldots, \tilde{c}_{m+k+1}\right\}$.

Selecting candidates $\left\{\tilde{c}_{m+1}, \ldots, \tilde{c}_{m+k+1}\right\}$ provides exactly $k$ representatives who agree with the voter majority on issues $\left\{s^{1}, \ldots, s^{r}\right\}$. Since we are selecting $2 k+1$ representatives in total, on any of these first $r$ issues we need only 1 more representative who agrees with the voter majority on each issue to achieve majority agreement. Therefore, selecting $k$ additional representatives from $\left\{\tilde{c}_{1}, \ldots, \tilde{c}_{m}\right\}$ which maximize coverage over issues $\left\{s^{1}, \ldots, s^{r}\right\}$, maximizes the majority agreement of the $2 k+1$ representatives over $\tilde{\mathcal{S}}$. These $k$ representatives are a one-to-one correspondence to the $k$ representatives in the solution to our original Max kCoverage problem.

Worse yet, even for small instances where the problem is computationally tractable, there are pathological examples for which truthful voters whose derived preferences over the candidates are perfectly consistent with their preferences over the issues will elect horrible representatives.

Theorem 4. No Condorcet-consistent election rule using approvals or total orderings can approximate Max k-Majority Agreement.

Our proof for Theorem 4 can be found in the full version of this paper [Abramowitz and Mattei, 2018] and is derived from an example found in [Anscombe, 1976] with 11 voters and 11 issues. This example is particularly pathological, because the worst candidate gets elected over the best candidate.

The difficulty of achieving majority agreement between the voters and candidates using the election alone - reinforced by our simulations below - and the existence of pathological cases even at small scales motivate the use of flexible delegations. However, as we saw from Example 1, delegations are not guaranteed to improve outcomes. We explore the potential benefits of such delegations in Section 5.

\section{Simulation Results}

We investigate the properties of coverage and majority agreement as a function of the numbers of candidates, issues, and committee size. In all our simulations, for all issues $s^{i}$ we let $v_{j}^{i}=1$ and $c_{l}^{i}=1$ with probability $\frac{1}{2}$ for all voters and candidates. This means that all candidates and voters come from the same populations, i.e., that they are both drawn from the same distribution; relaxing this assumption is an interesting direction for future work. In all of our runs, coverage was 1.0 for all combinations, hence we omit it from the graphs in Figure 1. For all simulations we perform 50 iterations at each datapoint and plot the mean $\left(\sigma^{2} \leq 0.002\right)$. For a first set of simulations we included rules that have NP-hard winner determination problems: CC and $k$-Median [Skowron, 2015]. We implemented these rules in Gurobi 8.1 and used a server with 16 cores and $32 \mathrm{~GB}$ of memory; taking nearly 24 hours to generate results for a smaller setting $(|\mathcal{C}|$ up to 17$)$. We found that both $\mathrm{CC}$ and $k$-Median are outperformed by Weights, STV, and AV at all settings and hence, we drop these rules in our larger analysis.

For our larger simulations we hold $|\mathcal{V}|=501$ fixed as we did not observe a strong dependence on the number of voters as long as it was sufficiently larger than the number of candidates. Turning first to Figure 1a we hold $|\mathcal{C}|=60,|k|=21$ and vary $|\mathcal{S}| \in\{15, \ldots, 150\}$ in steps of 15 . We see that for a small number of issues the AV, RAV, and the weighted voting rule can be expected to select a committee with very high majority agreement (0.8). However, as we add issues to the docket, the voting rules seem to converge around 0.6 . In Figure $1 \mathrm{~b}$ we fix $|k|=21,|\mathcal{S}|=150$ and vary the number of candidates between $|\mathcal{C}| \in\{21, \ldots, 100\}$ in steps of 5 . We observe again that AV, RAV, and weighted voting are the best followed closely by STV. As we increase the number of candidates it is possible for rules to select committees with higher majority agreement, but this number does not climb above $65 \%$. Finally, in Figure 1c we hold $|\mathcal{C}|=100,|\mathcal{S}|=150$ and vary $|k|$. These simulations reinforce the idea that electing an ideal committee, i.e., one that obtains perfect majority agreement, is a hard problem. In the next section we will explore how FRD can outperform RD and its dependence on the comparative use of delegations by the majority and minority.

\section{Benefits of Flexibility}

The flexibility of issue-specific delegations is the motivating feature of FRD. We look at basic features of FRD in a deterministic setting before considering probabilistic delegations.

\subsection{Deterministic Delegation}

A voter's delegation is incisive if the voter only delegates to representatives who agree with them on that issue. Observe that if the representatives are unanimous with no direct voting or dummy candidates to guarantee coverage only one outcome is possible, but as long as there is some dissent in the committee FRD can take advantage and return decision making power to the voters. 


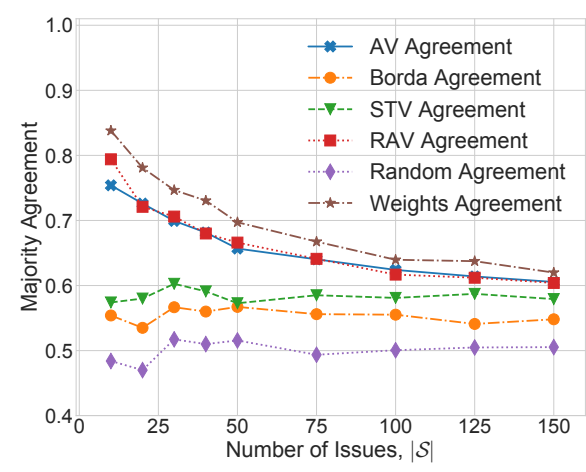

(a) Vary $|\mathcal{S}|$; fix $k=21,|\mathcal{C}|=60$.

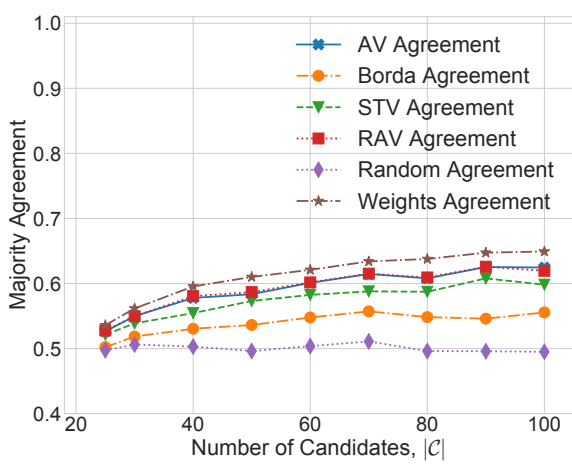

(b) Vary $|\mathcal{C}|$; fix $k=21,|\mathcal{S}|=150$.

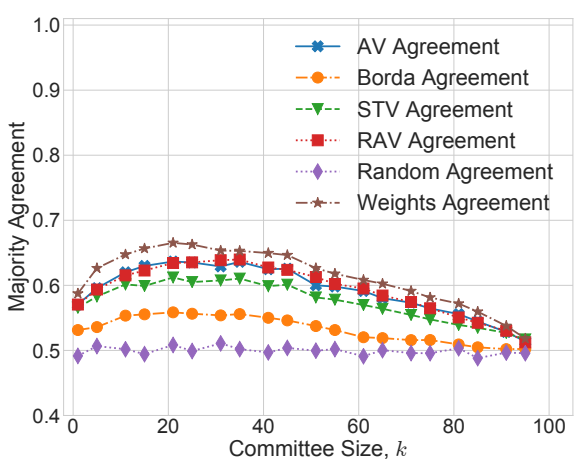

(c) Vary $k$; fix $|\mathcal{C}|=100,|\mathcal{S}|=150$.

Figure 1: Majority Agreement of the elected committee as a function of the number of issues, number of candidates, and committee size. Across all treatments the weighted voting, approval voting, and RAV select the best committees as measured by majority agreement.

We denote by $N_{1}^{i}+N_{0}^{i}=N$ the numbers of voters and by $k_{0}^{i}+k_{1}^{i}=k$ the numbers of candidates who agree and disagree with the voter majority on issue $s^{i}$, respectively. We have labeled the majority view of the voters as 1 , so $\forall s^{i} \in S: N_{1}^{i} \geq N / 2 \geq N_{0}^{i}$. The overall outcome of any resolute democratic process over this set of issues is an outcome vector $\mathcal{O}=\left\{\mathcal{O}^{1}, \ldots, \mathcal{O}^{r}\right\} \in\{0,1\}^{r}$, and our ideal outcome is $\{1\}^{r}$. Treating all issues equally and independently, we seek to maximize $\sum_{s^{i} \in S} \mathcal{O}^{i}$. Let $\lambda_{1}^{i}$ and $\lambda_{0}^{i}$ be the number of voters who delegate from the majority and minority respectively on issue $s^{i}$, assuming they delegate incisively. We drop the $i$ superscript below as we have a single issue.

Proposition 5. If all delegations are incisive and the representatives are not unanimous $\left(0<k_{1}<k\right)$, the outcome is guaranteed to agree with the voter majority if the number of voters in the majority who delegate $\left(\lambda_{1}\right)$ is greater than $\frac{N k-2 N_{1} k_{1}}{2\left(k-k_{1}\right)}$ and the outcome will favor the minority if $\lambda_{0}>\frac{k \lambda_{1}+\left(N-\lambda_{1}\right)\left(2 k_{1}-k\right)}{2 k_{1}}$.

\subsection{Probabilistic Delegation}

Here, instead of assuming that some fractions $\left(\alpha_{1}, \alpha_{0}\right)$ of voters delegate we investigate what happens if each voter chooses to delegate with some fixed individual probability. These results gives us an idea of how motivated or attentive voters must be to improve the outcome of FRD over RD. We assume that all voter and candidate preferences are independent for all issues.

As all issues are independent, we consider a single issue. Suppose each voter $v_{j} \in \mathcal{V}$ chooses to delegate (deviate from the default) with independent probability $p_{j}$ and that all delegations are incisive. Let $x_{j} \in[0,1]$ be the amount of power voter $v_{j}$ assigns to candidates who agree with the voter majority $\left(c_{l}=1\right)$, either by delegation or default. If $v_{j}$ defaults then $x_{j}=k_{1} / k$, if $v_{j}$ delegates incisively and is in the voter majority $\left(v_{j}^{i}=1\right)$ then $x_{j}=1$, and if $v_{j}$ delegates incisively and is in the voter minority then $x_{j}=0$. Let $X_{1}=\sum_{v_{j} \in \mathcal{V}} x_{j}$ be the total power assigned to these candidates via both delegation and default. Let $\mu=E\left[X_{1}\right]=$ $\sum_{v_{j}=1}\left(p_{j}+\left(1-p_{j}\right)^{k_{1} / k}\right)+\sum_{v_{j}=0}\left(1-p_{j}\right)^{k_{1} / k}$ be the expected value of the total power assigned to representatives who agree with the voter majority.

Theorem 6. Consider an FRD with an odd number of voters $N$, odd committee size $k$, and only incisive delegations. Suppose each voter $v_{j} \in \mathcal{V}$ delegates with probability $p_{j}$ on each issue such that $\mu>N / 2$. Then the probability that the outcome agrees with the voter majority is bounded by $P(\mathcal{O}=1) \geq 1-e^{-(N-2 \mu)^{2} / 4 N}$.

Proof. Recall that $x_{j} \in[0,1]$ is the amount of voting power voter $v_{j}$ assigns to candidates who agree with the voter majority on an issue and $X_{1}=\sum_{v_{j} \in \mathcal{V}} x_{j}$. Given some tie breaking rule, we have that $P(\mathcal{O}=1)=P\left(X_{1}>N / 2\right)+P(\mathcal{O}=$ $\left.1 \mid X_{1}=N / 2\right) \cdot P\left(X_{1}=N / 2\right)$. First we show that $P\left(X_{1}=\right.$ $N / 2)=0$, then we give a lower bound for $P\left(X_{1}>N / 2\right)$.

Lemma 1. If $N$ is odd, $k$ is odd, and all delegations are incisive, then no ties are possible.

Proof. Let $x_{j}^{\prime}=k \cdot x_{j}$ where $x_{j} \in[0,1]$ is the amount of weight (voting power) voter $v_{j}$ assigns to candidates who agree with the voter majority on an issue via default or delegation. If $v_{j}$ defaults then $x_{j}^{\prime}=k_{1}$, if $v_{j}$ delegates incisively and is in the voter majority $\left(v_{j}^{i}=1\right)$ then $x_{j}^{\prime}=k$, and if $v_{j}$ delegates incisively and is in the voter minority then $x_{j}^{\prime}=0$. Therefore, $\forall j: x_{j}^{\prime} \in\left\{0, k_{1}, k\right\}$. Let $X_{1}^{\prime}=\sum_{v_{j} \in \mathcal{V}} x_{j}^{\prime}$ and $X_{0}^{\prime}=\sum_{v_{j} \in \mathcal{V}}\left(k-x_{j}^{\prime}\right)$. Then $X_{1}^{\prime}, X_{0}^{\prime}$ are non-negative integers and $X_{1}^{\prime}+X_{0}^{\prime}=k N$. Since $k N$ is odd, it must be that $X_{1}^{\prime}$ and $X_{0}^{\prime}$ have opposite parity and so they cannot be equal. Therefore $X_{1}=X_{1}^{\prime} / k \neq X_{0}=X_{0}^{\prime} / k$, meaning the total amounts of weight delegated to the representatives on either side of the issue cannot be equal, so no ties may occur.

Given that no ties are possible, we have that $P(\mathcal{O}=$ $1)=P\left(X_{1}>N / 2\right)$. Remember that $X_{1}=\sum_{v_{j} \in \mathcal{V}} x_{j}$ where $x_{j}$ is the total weight that $v_{j}$ delegates to representatives who agree with the voter majority. If $v_{j}^{i}=1$ then $E\left[x_{j}\right]=p_{j}+\left(1-p_{j}\right) \frac{k_{1}}{k}$, else if $v_{j}^{i}=0$ then $E\left[x_{j}\right]=$ $\left(1-p_{j}\right) \frac{k_{1}}{k}$. Let $\mu=E\left[X_{1}\right]$ be the expected total weight assigned to representatives who agrees with the voter majority, then $\mu=\sum_{v_{j}^{i}=1}\left(p_{j}+\left(1-p_{j}\right) \frac{k_{1}}{k}\right)+\sum_{v_{j}^{i}=0}\left(1-p_{j}\right) \frac{k_{1}}{k}$. We 


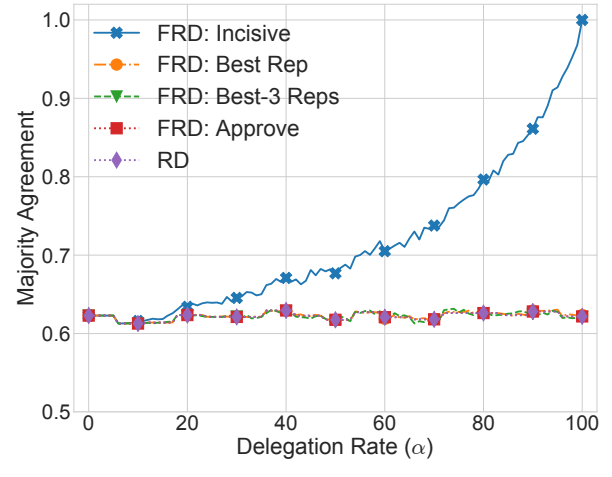

Figure 2: Majority agreement of the various systems with DD when the committee is selected using weighted voting.

now use the fact that $P\left(X_{1}>N / 2\right)=1-P\left(X_{1} \leq N / 2\right)$. Let $\delta=(2 \mu-N) / 2 \mu$. If $\mu>N / 2$, then $\delta>0$. This allows us to apply a Chernoff bound to derive our lower bound, $P\left(X_{1}>N / 2\right)=1-P\left(X_{1} \leq N / 2\right)=1-P\left(X_{1} \leq\right.$ $(1-\delta) \mu) \geq 1-e^{-\delta^{2} \mu^{2} / N}=1-e^{-(2 \mu-N)^{2} / 4 N}$.

This bound depends on the condition that $\mu>N / 2$. This assumption is only violated in rare cases when the minority is large, the majority delegates sparingly, and the representatives are near evenly split. As an increasing number of voters delegate incisively, we expect $\mu \rightarrow N_{1}>N / 2$ regardless of $k_{1}$. Naturally, as the delegation rate increases $(\alpha \rightarrow 1)$, we observe our lower bound approach the ideal $1-e^{-(2 \mu-N)^{2} / 4 N} \rightarrow 1$. To observe $\mu \leq N / 2$, the voter majority cannot be too large compared to the voter minority, $k_{1}$ must be smaller than or somewhat close to $k_{0}$, and/or the voters in the majority must be considerably more apathetic towards delegation than voters in the minority.

Tighter bounds may be achieved when the delegation probabilities are assumed to come from a particular distribution, or the preferences of voters and candidates are assumed to come from different distributions. It is an interesting open question to see how the expected outcome is effected when voters have different delegation probability distributions.

\subsection{Simulated Delegations}

We investigate the effect of the delegation rate $\alpha$ on the ability of different systems to recover the DD outcome, i.e., majority agreement. We use the same model to generate candidates and voter preferences as used in Section 4. For our simulated delegations we create instances with $|\mathcal{V}|=301,|\mathcal{C}|=60$, $|\mathcal{S}|=150$, and $k=21$. We vary $\alpha \in\{0,1.0\}$ in increments of 0.01 and for each setting of $\alpha$ we run 50 iterations. We plot means in Figure $2\left(\sigma^{2} \leq 0.002\right)$.

In Figure 2 we can see the majority agreement for the weighted voting committee selection rule for several delegation types and delegation rates. Note that a majority agreement of 1.0 means that the outcome of the system is identical to DD. We compare RD with four different delegation schemes: (1) in Approve voters delegate to the representatives of whom they approve and do not update; in Best Rep voters delegate to their single most preferred representative and do not update; in Best-3 Rep voters delegate equally to their three most preferred members of the committee and do not update; and finally in Incisive where voters delegate to a single representative with whom they agree per issue.

Most surprising is how little delegations that are not active and incisive help emulate DD. The Approve system is perhaps closest to the proposal of Proxy Voting espoused by Miller [1969] but does not improve RD in a meaningful way. Hence, we can see that the issue-specific flexibility FRD allows can be effectively used to achieve DD outcomes. Another striking result in Figure 2 is how drastically FRD can improve majority agreement over RD when voters are highly attentive. However, the high delegation rates required suggest that FRD may be burdensome to voters; exploring other delegation models is an important future direction.

\section{Conclusion}

We introduced a novel system called FRD which transitions smoothly, at the discretion of the voters, between direct and representative democracy. We have shown theoretically and empirically that FRD has the potential to overcome the shortcomings of other systems such as RD, LD, and Proxy Voting. An important point to remember is that in FRD, unlike in LD: delegations are optional, and not an additional burden imposed on the system or voters; in contrast to $\mathrm{LD}$, voters in FRD have greater certainty about how their vote will be cast ahead of time; and delegation cycles are not possible. Furthermore, FRD maintains a fixed, elected set of accountable representatives to produce legislation and hold public debates. This committee of representatives does not need to expand to guarantee proportional or justified representation. As Figure 2 demonstrates, the default distribution of voting power in FRD prevents a small subset of active voters from entirely determining the outcome as can happen in DD.

Our analysis makes best-case assumptions for RD: we assume all issues are known before the election, all voters participate in the election, candidate and representative preferences do not change, and voter preferences over candidates are consistent with their preferences over the issues. Relaxing any of these assumptions strengthens the argument for enabling flexible, issue-specific delegations. Flexible delegation also minimizes the role that election rule plays in the outcome. Extensions and future work including more realistic voter models, different candidate models, incorporating aspects of judgement aggregation including removing the independence between issues, and different committee decision rules, to name just a few, see Abramowitz and Mattei [2018].

\section{Acknowledgments}

Much of this work was completed while both authors were working at IBM Research AI, Yorktown Heights, NY. We would like to thank Nick Dalmasso and the anonymous reviewers for their guidance and input.

\section{References}

[Abramowitz and Mattei, 2018] B. Abramowitz and N. Mattei. Flexible representative democracy: An introduction with binary issues. arXiv preprint arXiv:11811.02921, 2018. 
[Alger, 2006] D. Alger. Voting by proxy. Public Choice, 126(1/2):1-26, 2006.

[Anscombe, 1976] G. E.M. Anscombe. On frustration of the majority by fulfilment of the majority's will. Analysis, 36(4):161-168, 1976.

[Auriol and Gary-Bobo, 2012] E. Auriol and R. J. Gary-Bobo. On the optimal number of representatives. Public Choice, 153(34):419-445, 2012.

[Aziz et al., 2017] H. Aziz, M. Brill, V. Conitzer, E. Elkind, R. Freeman, and T. Walsh. Justified representation in approvalbased committee voting. Social Choice and Welfare, 48(2):461485, 2017.

[Baharad et al., 2012] E. Baharad, J. Goldberger, M. Koppel, and S. Nitzan. Beyond Condorcet: Optimal aggregation rules using voting records. Theory and decision, 72(1):113-130, 2012.

[Banzhaf III, 1964] J. F. Banzhaf III. Weighted voting doesn't work: A mathematical analysis. Rutgers L. Rev., 19:317, 1964.

[Ben-Yashar and Nitzan, 1997] R. C. Ben-Yashar and S. I. Nitzan. The optimal decision rule for fixed-size committees in dichotomous choice situations: The general result. International Economic Review, pages 175-186, 1997.

[Bloembergen et al., 2018] D. Bloembergen, D. Grossi, and M. Lackner. On rational delegations in liquid democracy. arXiv preprint arXiv:1802.08020, 2018.

[Blum and Zuber, 2016] C. Blum and C. I. Zuber. Liquid democracy: Potentials, problems, and perspectives. Journal of Political Philosophy, 24(2):162-182, 2016.

[Brandt et al., 2016] F. Brandt, V. Conitzer, U. Endriss, J. Lang, and A. D. Procaccia, editors. Handbook of Computational Social Choice. Cambridge University Press, 2016.

[Brill and Talmon, 2018] M. Brill and N. Talmon. Pairwise liquid democracy. In Proc. 27th IJCAI, pages 137-143, 2018.

[Brill, 2018] M. Brill. Interactive democracy. In Proc. 17th AAMAS, pages 1183-1187, 2018.

[Christoff and Grossi, 2017] Zoé Christoff and Davide Grossi. Binary voting with delegable proxy: An analysis of liquid democracy. In Proc. 16th TARK, pages 134-150, 2017.

[Cohensius et al., 2017] G. Cohensius, S. Mannor, R. Meir, E. A. Meirom, and A. Orda. Proxy voting for better outcomes. In Proc. 16th AAMAS, pages 858-866, 2017.

[Domshlak et al., 2011] C. Domshlak, E. Hüllermeier, S. Kaci, and H. Prade. Preferences in AI: An overview. AI, 175(7):1037-1052, 2011.

[Dunn, 1995] J. Dunn. Democracy: The Unfinished Journey. Oxford University Press, 1995.

[Feige, 1998] U. Feige. A threshold of $\ln n$ for approximating set cover. Journal of the ACM, 45(4):634-652, 1998.

[Feld and Grofman, 1984] S. L. Feld and B. Grofman. The accuracy of group majority decisions in groups with added members. Public Choice, 42(3):273-285, 1984.

[Garey and Johnson, 1979] M. R. Garey and D. S. Johnson. Computers and Intractability, A Guide to the Theory of NPCompleteness. W.H. Freeman and Company, 1979.

[Green-Armytage, 2015] J. Green-Armytage. Direct voting and proxy voting. Constitutional Political Economy, 26(2):190-220, 2015.
[Grofman and Feld, 1983] B. Grofman and S. L. Feld. Determining optimal weights for expert judgment. In Information Pooling and Group Decision Making, pages 167-72, 1983.

[Grofman et al., 1983] B. Grofman, G. Owen, and S. L. Feld. Thirteen theorems in search of the truth. Theory and Decision, 15(3):261-278, 1983.

[Hardt and Lopes, 2015] S. Hardt and L. C. Lopes. Google votes: A liquid democracy experiment on a corporate social network. Technical Disclosure Commons, 2015.

[Kahng et al., 2018] A. Kahng, S. Mackenzie, and A. D Procaccia. Liquid democracy: An algorithmic perspectiven. In Proc. 32nd AAAI, 2018.

[Karotkin and Paroush, 2003] Drora Karotkin and Jacob Paroush. Optimum committee size: Quality-versus-quantity dilemma. Social Choice and Welfare, 20(3):429-441, 2003.

[List and Puppe, 2009] C. List and C. Puppe. Judgment aggregation: A survey. In C. List and C. Puppe, editors, Handbook of Rational and Social Choice, pages 457-482. Oxford University Press, 2009.

[Magdon-Ismail and Xia, 2018] M. Magdon-Ismail and L. Xia. A mathematical model for optimal decisions in a representative democracy. arXiv preprint arXiv:1807.06157, 2018.

[Miller, 1969] J. C. Miller. A program for direct and proxy voting in the legislative process. Public Choice, 7(1):107-113, 1969.

[Nitzan and Paroush, 1982] S. Nitzan and J. Paroush. Optimal decision rules in uncertain dichotomous choice situations. International Economic Review, pages 289-297, 1982.

[Nitzan and Paroush, 2017] S. Nitzan and J. Paroush. Collective decision making and jury theorems. Oxford Handbook of Law and Economics, pages 494-516, 2017.

[Paroush and Karotkin, 1989] J. Paroush and D. Karotkin. Robustness of optimal majority rules over teams with changing size. Social Choice and Welfare, 6(2):127-138, 1989.

[Rodriguez and Steinbock, 2004] M. A. Rodriguez and D. J. Steinbock. Societal-scale decision making using social networks. arXiv preprint arXiv: cs/0412047, 2004.

[Shapley and Shubik, 1954] L. S. Shapley and M. Shubik. A method for evaluating the distribution of power in a committee system. American political science review, 48(3):787-792, 1954.

[Shubik, 1970] M. Shubik. On homo politicus and the instant referendum. Public Choice, 9:79-84, 1970.

[Skowron et al., 2016] P. Skowron, P. Faliszewski, and J. Lang. Finding a collective set of items: From proportional multirepresentation to group recommendation. AI, 241:191-216, 2016.

[Skowron, 2015] P. Skowron. What do we elect committees for? A voting committee model for multi-winner rules. In Proc. 24th IJCAI, pages 1141-1147, 2015.

[Smith, 1973] J. H. Smith. Aggregation of preferences with variable electorate. Econometrica: Journal of the Econometric Society, pages 1027-1041, 1973.

[Tullock, 1967] G. Tullock. Toward a mathematics of politics. University of Michigan Press, 1967.

[Tullock, 1992] G. Tullock. Computerizing politics. Mathematical and Computer Modelling, 16(8-9):59-65, 1992.

[Yu et al., 2010] C.-H. Yu, J. Werfel, and R. Nagpal. Collective decision-making in multi-agent systems by implicit leadership. In Proc. 9th AAMAS, pages 1189-1196, 2010. 